\title{
Ambiente físico-social y envejecimiento de la población desde la gerontología ambiental y geografía. Implicaciones socioespaciales en América Latina ${ }^{1}$
}

\author{
Diego Sánchez González²
}

\begin{abstract}
RESUMEN
El estudio reflexiona sobre el análisis de los ambientes físico-sociales que determinan la calidad de vida de las personas mayores en América Latina desde el enfoque de la gerontología ambiental. A través de la literatura, se favorece la discusión sobre las implicaciones socioespaciales urbanas y rurales en las dimensiones residenciales (entorno construido), sociales, económicas, de salud, asistenciales (servicios sociales) y culturales, y que promueven el envejecimiento saludable en el lugar. También, se abordan las principales cuestiones teóricas y metodológicas de las implicaciones socioespaciales para la gerontología ambiental, donde destaca la contribución de la geografía, a través de una revisión de las complejas relaciones entre el espacio geográfico y las personas adultas mayores. Así, en la región existe la necesidad de un enfoque interdisciplinario que enfatice la investigación sobre el análisis de los atributos y funciones del entorno físico-social en el envejecimiento de la población, contribuyendo a la planificación gerontológica desde una perspectiva holística.
\end{abstract}

Palabras clave: Ambiente físico-social, calidad de vida, gerontología ambiental, geografía del envejecimiento, América Latina.

\begin{abstract}
The study reflects on the analysis of the physical-social environments that determine the quality of life of seniors in Latin America from the approach of environmental gerontology. Through the literature, we develop a discussion of urban and rural sociospatial implications on the residential dimensions (built environment), social, economic, health, welfare (social services) and cultural, and to promote healthy aging in place. Also addressed are the main theoretical and methodological issues of the socio-spatial implications for environmental gerontology, which highlight the contribution of geography, through a review of the complex relationships between geographical space and the elderly. Thus, in the region there is a need for an interdisciplinary approach that emphasizes research on the analysis of the attributes and functions of the physical-social environment in the aging population, contributing to the gerontological planning from a holistic perspective.
\end{abstract}

Key words: Physical-social environment, quality of life, environmental gerontology, geography of aging, Latin America.

\footnotetext{
Este trabajo se deriva de un proyecto de investigación "Gerontología ambiental del envejecimiento vulnerable en áreas de riesgo a inundaciones. Retos de la gestión de los riesgos y la planificación gerontológica ante el cambio climático" ( $\mathrm{N}^{\circ}$ 155757), Consejo Nacional de Ciencia y Tecnología (CONA-
}

CYT), México. Artículo recibido el 17 de septiembre de 2013, aceptado el 9 de septiembre de 2014 y corregido el 20 de octubre de 2014.

2 Facultad de Arquitectura, Universidad Autónoma de Nuevo León (México).

E-mail: diego.sanchezgn@uanl.edu.mx 
En la década de 1970 se creía que la longevidad estaba determinada por factores genéticos (Gutiérrez, 2010). Cuatro décadas más tarde se constata que el contexto ambiental, tanto físico-construido como social, explica en mayor medida la esperanza de vida, la longevidad y la propensión a desarrollar patologías relacionadas con la edad (alzheimer, cáncer) (Fetter et al., 2012).

Hoy estamos empezando a comprender que a través del control del ambiente se puede influir en el bienestar físico y emocional de las personas adultas mayores. La calidad de vida de la población que envejece está sujeta a las múltiples negociaciones que se establecen entre los heterogéneos sujetos y sus entornos. Al respecto, los expertos coinciden en el diseño de políticas sociales sostenibles enfocadas al proceso de envejecer en el lugar $\mathrm{u}$ hogar (aging in place) (Andrews \& Phillips, 2005). Sin embargo, surgen importantes interrogantes sobre los problemas a los que se enfrentan los adultos mayores en sus entornos cotidianos; así como el modelo a seguir para construir entornos físicos y sociales propicios para envejecer en el lugar. Asimismo, los profesionales (gerontólogos, geógrafos, urbanistas) deben conocer el reto de planificar espacios para una población que envejece en un contexto de cambio climático.

Desde diversas disciplinas de las ciencias sociales, como geografía y psicología, se han realizado aportaciones para comprender la importancia del entorno físico-construido y entorno social en la experiencia espacial de las personas que envejecen (Lawton, 1990; Sánchez-González, 2009a). De esta forma, estamos comenzando a entender las experiencias positivas y negativas de las personas mayores en relación con su entorno cotidiano (urbano y rural), desde perspectivas objetivas y subjetivas, analizando la habitabilidad y las desigualdades ambientales.

La revisión de la literatura confirma la relación entre el avance del envejecimiento demográfico de los países desarrollados (Estados Unidos, Canadá, Reino Unido, Alemania, Australia, Japón) y el auge de las cuestiones ambientales del envejecimiento en las ciencias sociales, como geografía, lo que ha propiciado el desarrollo de la gerontología am- biental ${ }^{3}$. Entre los estudios pioneros de esta rama de la gerontología destaca la importancia de la relación entre los distintos contextos ambientales y las heterogéneas capacidades de adaptación de las personas adultas mayores, mostrando un especial interés por revelar su incidencia en la conducta (Lawton \& Nahemow, 1973). Precisamente, se indica que las personas envejecidas negocian con su entorno físico-social, tratando de encontrar un equilibrio entre sus capacidades (progresivamente mermadas con la edad) y las presiones del ambiente (Lawton, 1985 y 1990; Stokols, 1995; Shigematsu et al., 2009), el cual, debe ser analizado a través de distintas escalas geográficas: escala macro, región y ciudad; escala meso, barrio, y escala micro, la vivienda o residencia y su espacio personal.

Las cuestiones ambientales del envejecimiento de la población están adquiriendo relevancia en la literatura geográfica anglosajona, lo que ha propiciado el surgimiento de la geografía del envejecimiento o gerontológica4 ${ }^{4}$ (Warnes, 1990; Sylvestre, 1999). A partir de los años 1970 en los países anglosajones se va configurando la geografía del envejecimiento centrada en el análisis de las implicaciones socioespaciales del envejecimiento de la población y la comprensión de las complejas relaciones entre el entorno físico-social y las personas mayores a distintas escalas (Rowles, 1978). Así, se va destacando la contribución de las cuestiones geográficas a la gerontología y, especialmente, la gerontología ambiental, al reconocerse que el espacio geográfico afecta al proceso de envejecimiento, realizándose estudios centrados en los patrones espaciales del envejecimiento de la población asociados con su movili-

\footnotetext{
3 La gerontología ambiental es una área de conocimiento de la gerontología que tiene por objetivo conocer, analizar, modificar y optimizar la relación entre la persona que envejece y su entorno físico-social, desde perspectivas y enfoques interdisciplinarios, que abarcan disciplinas como geografía, psicología, arquitectura, diseño, urbanismo, ciencias de la salud, trabajo social, sociología y otras ciencias a fines (Wahl \& Weisman, 2003; Rowles \& Bernard, 2013).

4 La geografía del envejecimiento se centra en el estudio de las complejas relaciones entre el espacio geográfico y las personas mayores, dentro de un contexto socioespacial integrado por los entornos físico-construido y humano-social.
} 
dad residencial y el acceso a los servicios sociales (Harper \& Laws, 1995; Golant \& Salmon, 2004; Rowles \& Chaudhury, 2005). Así, se constata el desarrollo de perspectivas tradicionales y enfoques cuantitativos de las dinámicas de distribución del envejecimiento demográfico asociadas a las cuestiones residenciales, de salud y servicios sociales; así como nuevas perspectivas y enfoques cualitativos vinculados al estudio de las complejas relaciones entre los heterogéneos entornos residenciales y asistenciales, y las personas mayores (Sánchez-González, 2011; Andrews et al., 2007).

En América Latina el estudio del envejecimiento de la población está ligado a los conceptos de edad, vejez y envejecimiento, que son producto de una construcción social que varía entre regiones, grupos sociales y culturas, y que tiene importantes implicaciones socioespaciales para una región heterogénea y determinada por grandes desigualdades sociales (Chackiel, 2000). También, el avance de las políticas sociales, salud, pensiones y servicios sociales, está contribuyendo a aumentar la esperanza de vida de la población más allá de los 65 años (Trujillo et al., 2007; Ham-Chande et al., 2009; Díaz-Tendero, 2011). Asimismo, se estima que el aumento del envejecimiento demográfico implicará adecuaciones ambientales para atender las crecientes necesidades y demandas de servicios, equipamientos e infraestructuras destinados a los millones de adultos mayores vulnerables (pobreza, desatención, soledad, violencia) (Sánchez-González, 2007). A pesar del avance en materia de políticas públicas, muy poco se conoce del verdadero impacto que los diferentes entornos físico-sociales ${ }^{5}$ (urbanos y rurales) tendrán en el envejecimiento de la población en la región.

El artículo reflexiona sobre el análisis de los ambientes físico-sociales que determinan la calidad de vida de las personas mayores en América Latina, desde el enfoque de la gerontología ambiental. Del mismo modo, se abordan las principales cuestiones teóricas y

\footnotetext{
5 El término entorno físico-social procede de la psicología ambiental y se refiere a la complejidad del medio ambiente y sus interrelaciones físicas, sociales, culturales y organizacionales.
}

metodológicas de esta rama de la gerontología, donde destaca la geografía, asociadas al análisis del espacio geográfico urbano y rural, con objeto de promover el envejecimiento saludable en el lugar. Además, se enfatiza la necesidad de estudios interdisciplinares en el análisis de los atributos y funciones del ambiente en el envejecimiento, y sus implicaciones socioespaciales en la planificación gerontológica.

\section{Ambiente, calidad de vida y envejecimiento de la población en América Latina}

En América Latina se ha prestado escasa atención a las crecientes implicaciones socioespaciales del envejecimiento de la población, inicialmente achacable al menor envejecimiento demográfico y a problemas estructurales de carácter organizativo y económico, que favorecen la falta de sensibilidad y planificación hacia esta cuestión central, que está desbordando la limitada capacidad de respuesta de los gobiernos. De la misma forma, se ha producido un cambio de perspectiva en las políticas públicas sobre envejecimiento, desde insostenibles programas de institucionalización hacia nuevos enfoques vinculados al envejecimiento en el lugar, lo que obliga a prestar especial atención al entorno físico-social donde se desarrolla la vida cotidiana del adulto mayor.

El desigual avance del envejecimiento de la población en la región ${ }^{6}$, como fenómeno geográfico generalizado y heterogéneo, está relacionado con el proceso de transición demográfica, y determinado, principalmente, por la caída de la fecundidad, el descenso de la mortalidad y los movimientos migratorios. Al respecto, los estudios advierten sobre el reto del envejecimiento que deben enfrentar los gobiernos de la región, ya que se prevé que entre los años 2013 y 2050 su población de 60 años y más se triplicará, pasando de 65,5 a 195,9 millones de personas, mientras

\footnotetext{
6 En el año 2013 los países con las mayores tasas de población de 60 y más años son Puerto Rico $(18,7 \%)$, Uruguay $(18,4 \%)$ y Cuba $(18,3 \%)$, mientras que Belize $(5,7 \%)$, Honduras $(6,4 \%)$ y Guatemala $(6,5 \%)$ presentan las tasas más bajas (ONU, 2013).
} 
que su peso relativo se incrementará del 10,6 al 25,1\% (ONU, 2013). En la misma línea, los expertos asocian las proyecciones del fenómeno al actual contexto de pobreza, inequidad y baja cobertura de los sistemas de seguridad social para los adultos mayores, así como la necesidad de revisar las políticas públicas vinculadas al mercado laboral, pensiones, servicios sociales y de salud, y vivienda (Guzmán, 2002; López-Salgado, 2006; Huenchuan, 2009).

Las investigaciones han permitido conocer la desigual distribución del envejecimiento de la población a distintas escalas geográficas en la región, configurando un modelo polarizado basado en la concentración urbana y la dispersión rural. Este hecho convierte el envejecimiento demográfico urbano ${ }^{7}$ en un hecho sin precedentes y problemático, lo que debe favorecer el estudio de las ciudades por gerontólogos ambientales y, especialmente geógrafos. A su vez, el análisis de las implicaciones socioespaciales del fenómeno está contribuyendo a la comprensión de las diferencias de calidad de vida de los adultos mayores, y a favorecer una mejor planeación gerontológica de las políticas públicas para abatir la desigualdad y potenciar el bienestar de la población de 60 y más años a nivel nacional, estatal y municipal, así como en entornos urbanos y rurales (Sánchez-González, 2007). Así, en el análisis de los factores determinantes de la desigual distribución y localización de la población envejecida adquieren relevancia los patrones de movilidad y migración en el envejecimiento (vivienda, salud, dependencia, turismo). De hecho, el factor migratorio explica en gran medida las diferencias interurbanas $y$, sobre todo, intraurbanas del fenómeno (Jasso et al., 2011), como en Ciudad de México (Negrete, 2003) y La Habana (Bello, 2013). Como testimonio de ello, buena parte de la población anciana

\footnotetext{
7 En el año 2010 la distribución relativa de la población de 60 y más años de las principales urbes de la región indica que Montevideo* (26\%), Buenos Aires $(21,7 \%)$ y La Habana $(19,9 \%)$ registran las tasas más elevadas. También, en valores medios encontramos a Río de Janeiro (13,3\%), São Paulo $(10,7 \%)$, Santiago $(12,7 \%)$, Ciudad de Panamá $(10 \%)$ y Ciudad de México (9,9\%). A su vez, con los valores más bajos destacan Quito $(9,3 \%)$ y Guayaquil $(8,4 \%)$ (ONU, 2013). (*) Datos del año 2011.
}

que hoy reside en las ciudades ha nacido en las zonas rurales, como en Río Grande do Sul (Brasil) (CEl, 1997), lo que dificulta los procesos de adaptación al medio en la vejez.

Entre las temáticas de movilidad residencial y estrategias residenciales de las personas de edad son reseñables las aportaciones sobre las motivaciones (personales, familiares, laborales) y sus efectos en la estructura familiar (Gomes da Conceiqáo, 1997; Caprón y González, 2010). También, se ha abordado cómo el cambio de lugar de residencia puede implicar un proceso traumático para el adulto mayor al alejarse de su entorno cotidiano. Así, se ha constatado que la nostalgia y el anhelo de regresar tienen efectos negativos sobre la salud del adulto mayor migrante, como los jubilados puertorriqueños en Estados Unidos (Todorova et al., 2014). En las regiones rurales la permanencia de la población anciana se ha visto incrementada por la llegada de emigrantes de retorno de edades avanzadas a sus lugares de origen (Sánchez-González, 2007). De igual forma, se está abordando la migración de retorno y sus efectos sobre la vulnerabilidad de los adultos mayores retornados y la débil capacidad de respuesta de los gobiernos locales, como en la frontera de México y Estados Unidos (Chávez y SánchezGonzález, 2012).

La migración asociada al turismo de la tercera edad está teniendo una importancia creciente para las economías locales, como Río Claro (Brasil) (Balsan, 2006); las ciudades costeras de Mazatlán y Cabo San Lucas (México), donde este colectivo participa como inversor inmobiliario y actor urbano de la planificación (Lizárraga, 2008; Kiy \& McEnany, 2010); las ciudades históricas de la América Andina (Arequipa, Cuenca) (Hayes, 2013); y los espacios rurales de Tamaulipas (México) (Sánchez-González, 2008). De las investigaciones se desprende la falta de fiabilidad de los registros para cuantificar el fenómeno, lo que puede estar asociado a factores como el desinterés y desconfianza de este sector de población.

En los últimos años se está prestando especial atención al ambiente y su relación con el envejecimiento activo y saludable en el lugar. Desde este nuevo enfoque adquieren relevancia el análisis de las implicaciones 
socioespaciales del fenómeno, y la comprensión de las complejas relaciones del entorno físico-social y el adulto mayor, donde se advierten las diferencias significativas entre las presiones ambientales y las estrategias de adaptación de los individuos longevos. Precisamente, recientes estudios indican que la vulnerabilidad de este colectivo está asociada a factores biológicos, sociales y, sobre todo, ambientales, vinculados a la discapacidad, dependencia y exclusión social, así como a los peligros naturales y antrópicos (SánchezGonzález y Egea, 2011).

En América Latina el peso relativo de la población de 60 y más años es más acusado en las áreas rurales, vinculado a la fuerte emigración. También, se constata la existencia de una mayor vulnerabilidad de los adultos mayores en las zonas rurales que en las zonas urbanas, que afecta sobre todo a mujeres y personas de 75 y más años, debido a sus bajos niveles educativos (analfabetismo), bajos ingresos (pobreza), aislamiento, precariedad de la vivienda, problemas de acceso a los servicios sociales y de salud, limitación en transportes públicos, así como la ausencia de políticas públicas enfocadas a este colectivo. Esta circunstancia se ve agravada por la mayor prevalencia de problemas de salud (diabetes mellitus, hipertensión arterial) y la existencia de altas tasas de dependencia vinculadas al mayor coste para acceder a los escasos e inaccesibles servicios de salud, como en los municipios rurales de los estado de Oaxaca y Tamaulipas (México) (SánchezGonzález, 2007 y 2008), y en las zonas cafetaleras de Colombia (Gómez y Curcio, 2004). Lo cual, se traduce en una menor esperanza de vida en el medio rural, circunstancia que se agudiza entre la población adulta mayor indígena. Esta realidad convierte al entorno rural en un medio problemático para envejecer en el lugar escasamente abordado, por lo que es necesario favorecer nuevos estudios sobre estos complejos ambientes físicosociales y contribuir al desarrollo de políticas sociales.

En un contexto de progresivo envejecimiento demográfico urbano adquieren enorme importancia las implicaciones socioespaciales del espacio urbano sobre la calidad de vida del adulto mayor. Sin embargo, las ciudades de la región no favorecen el envejecimiento en el lugar, derivado del rápido proceso urbanizador no planeado y las desigualdades sociales (Formiga y Prieto, 2010; Sánchez-González, 2013), caso de las ciudades brasileñas (Jacomini, 1990), mexicanas (Serrano et al., 2009) y uruguayas (Guidotti y Aidar, 2012). Al respecto, los procesos especulativos y de marginación, asociados a la desigual distribución de la oferta de servicios y equipamientos, así como el deterioro medioambiental, transforman el entorno urbano en un medio hostil para envejecer (Jorge, 2009; Narváez, 2012). Diferentes estudios geográficos han abordado la morbilidad y mortalidad de la población envejecida asociadas a los efectos del ambiente urbano (Pizarro, 2005), como las muertes por causas externas vinculadas a la contaminación atmosférica (tráfico, industrias), y al acceso al agua potable y al saneamiento, como en São Paulo (Saldiva et al., 1995; Mathias et al., 2006). También, se ha comprobado las implicaciones del espacio urbano en el riesgo de caídas, atropellos y delincuencia, así como problemas psicológicos (depresión, ansiedad, estrés) entre los adultos mayores (Peixoto et al. 2008), lo que favorece el confinamiento ${ }^{8}$ en sus domicilios, con efectos negativos sobre su calidad de vida (relaciones sociales, ocio, utilización de servicios sociales y de salud) (Mejía et al., 2007; Tapia et al., 2010). Precisamente, los barrios históricos demográficamente envejecidos, como en Bahía Blanca, presentan altas tasas de personas mayores en situaciones de aislamiento y exclusión social (Bagnulo y Pizarro, 2010). Todo ello confirma que las ciudades latinoamericanas contribuyen a incrementar la vulnerabilidad de la heterogénea población envejecida (diferencias según cohorte y género), amenazada por procesos biológicos y sociales, como la merma en la salud y los ingresos; así como expuesta a peligros naturales y antrópicos. En este sentido, las soluciones gubernamentales brindadas a la fecha han sido parciales (tipologías de viviendas no vinculadas a la

\footnotetext{
8 Algunos expertos (Jacobson, 2011) defienden que el confinamiento en la vejez puede estar relacionado con enfermedades, como agorafobia e hipocondría, sosteniendo que no son solo problemas psicológicos, sino trastornos asociados a la inadaptación de las personas mayores, sobre todo mujeres, a los espacios urbanos y situaciones estresantes que los rodean.
} 
satisfacción residencial, espacios públicos no planeados, servicios sociales inaccesibles) y alejadas de las necesidades de la población envejecida. Además, se desprende la importancia de contar con registros (censales, hospitalarios) más confiables, así como análisis socioespaciales más precisos de las características del entorno urbano donde se envejece.

Hoy las ciudades se han convertido en los principales ambientes de estudio, siendo esenciales en el diseño de políticas públicas locales destinadas a este colectivo. En los últimos años la agenda internacional subraya la importancia de favorecer ciudades amigables con las personas de edad ${ }^{9}$ (OMS, 2007). Esta iniciativa gubernamental plantea la necesidad de un mayor conocimiento de las cuestiones ambientales asociadas a la vejez (espacios al aire libre y edificios, transportes, vivienda, participación social, respeto e integración social, participación cívica y empleo, comunicación e información, y apoyo de la comunidad y servicios de salud), desde nuevos enfoques cuantitativos y cualitativos. Así, el enfoque ecológico defiende un urbanismo saludable que contribuya al éxito de los procesos de envejecimiento (Silveira, 2003; Tomasini, 2005), a través de la reducción de la carga ambiental y la mejora de la capacidad funcional de la persona de edad para hacer frente a los cambios del medio. En este sentido, se están analizando las características del ambiente urbano que favorecen la calidad de vida del adulto mayor, como los equipamientos y la seguridad del barrio, así como su participación en organizaciones, caso de Valparaíso (Chile) (Fadda y Cortés, 2009).

\footnotetext{
9 En el año 2005 la Organización Mundial de la Salud (OMS, 2007) inicia el proyecto de ciudades amigables con las personas de edad, basado en un acuerdo de recomendaciones, y al que se sumaron 33 ciudades de todo el mundo, de las cuales, una cuarta parte de ellas se localizan en América Latina y el Caribe, como La Plata, Río de Janeiro, San José de Costa Rica, Kingston, Montego Bay, Cancún, Ciudad de México, Mayagüez y Ponce. Tras nueve años, el proyecto ha tenido un desigual avance en la región, marcado por los desequilibrios regionales y la desigualdad social, así como las diferencias en políticas sociales, y grado de cohesión social y de reivindicación del grupo etario.
}

Recientes estudios subrayan la importancia de la experiencia espacial vinculada al espacio simbólico de la vida cotidiana, el apego e identidad de lugar en el envejecimiento, donde la vivienda y el vecindario (urbano y rural), como espacios de la esfera privada y pública, adquieren gran relevancia en el bienestar físico-psicológico, por sus conexiones con la espiritualidad y el sentido de la historia del adulto mayor (SánchezGonzález, 2014). Desde la geografía de la percepción se indica que para favorecer un lugar para envejecer, se debe optimizar el ambiente a partir de la percepción de las estructuras que garanticen el bienestar, y de la comprensión del proceso de pertenencia a un espacio simbólico marcado por las experiencias, recuerdos y estigmas (Fatiga, 2012).

Las nuevas políticas de salud se interesan en la mejora de los entornos físico-sociales, ya que, favorecen los estilos de vida saludables y la calidad de vida del adulto mayor. Así, se enfatiza la relevancia del paisaje ${ }^{10}$ y los espacios públicos ${ }^{11}$, al potenciar los estilos de vida saludables, la recreación y las conexiones sociales locales (relaciones sociales), que posibilitan la cohesión de las comunidades que envejecen. Al respecto, se indica que favorecer la atención y acceso a los espacios públicos (parques, áreas verdes), propicia las actividades al aire libre (caminar), las relaciones sociales y el envejecimiento saludable en el lugar (Péricles et al., 2009; Cao, 2010; Salas y Sánchez-González, 2014). Precisamente, se ha observado una relación significativa entre la salud sexual de los adultos mayores y la proximidad del vecindario a parques públicos seguros, y de manera inversa con el ruido en la calle y la inseguridad ciudadana, como en Bogotá (Parra et al., 2010).

De todo ello, se deduce la necesidad de considerar en los estudios gerontológicos y

\footnotetext{
10 Gastaldo et al. (2004) subraya la importancia del valor estético y terapéutico del paisaje sobre la salud y la satisfacción residencial de las personas mayores.

${ }^{11}$ La geógrafa Sheila Peace et al. (2006) advierten de la importancia de construir espacios públicos para favorecer el envejecimiento en el hogar, desde una perspectiva holística de las relaciones sociales, y a través de generar lugares amigables, independientemente de la edad, salud y cultura.
} 
geográficos propuestas tangibles para favorecer ambientes urbanos y rurales amigables con las personas mayores de la región, a partir del análisis de las adecuaciones ambientales específicas (residenciales, servicios sociales y de salud) a las necesidades y comportamientos individuales y colectivos. Al respecto, los adultos mayores son portadores de funciones vitales y procesos sociales en los espacios urbanos y rurales, y la modificación de sus comportamientos espaciales por el contexto ambiental y sociocultural (valoraciones y actuaciones) implica cambios en las funciones y características de los ambientes, por lo que es imprescindible que participen activamente en la planificación urbana ${ }^{12}$. En la misma línea, la comprensión de las interacciones socioespaciales y del comportamiento de la población envejecida debe estar en el germen de las futuras políticas públicas.

\section{Desafíos teóricos y metodológicos en el análisis del ambiente en el envejecimiento}

El presente de la gerontología ambiental no está exento de polémica, propia de un campo multidisciplinar relativamente reciente y desconocido para el gran público. En este apartado, discutimos sobre los principales problemas teóricos y metodológicos planteados en el análisis de los contextos ambientales del envejecimiento, con alusiones a América Latina.

En la actualidad, diferentes expertos (Wahl \& Weisman, 2003) ponen en duda algunas de las principales teorías de la gerontología ambiental ${ }^{13}$, como el modelo ecológico

\footnotetext{
12 Las experiencias cartográficas a través de la educación geográfica entre las personas mayores pueden propiciar su participación y la formulación de propuestas para mejorar su entorno (Duarte et al., 2009).

${ }^{13}$ En las últimas décadas en la gerontología ambiental han coexistido diferentes modelos teóricos (Fernández-Trocóniz y Fernández-Ballesteros, 1990), derivados y/o críticos con el modelo ecológico de la competencia (Lawton \& Nahemow, 1973), como el modelo de la congruencia y el modelo integrador (Carp \& Carp, 1984).
}

de la competencia ${ }^{14}$ (Lawton \& Nahemow, 1973), que defendía el determinismo ambiental y presentaba limitaciones de aplicabilidad en casos concretos, la vivienda y el entorno institucionalizado. Asimismo, nos sumamos a dichas críticas, por considerar que en la teoría se ignoran las relaciones positivas entre las personas mayores y el medio ambiente, así como los comportamientos proactivos de las individuos longevos como agentes de cambio de sus ambientes ${ }^{15}$, sin olvidar, la variedad de entornos físicos-sociales (barrio y ciudad) y los nuevos entornos inteligentes y virtuales (internet), determinantes en el envejecimiento en el lugar. Al respecto, el geógrafo Stephen Golant (2011) defiende que, a pesar de los desafíos ambientales (viviendas no adaptadas, barrios marginados, cambio de residencia), las personas mayores pueden encontrar recursos personales para negociar con su entorno cotidiano y mantener el bienestar.

A pesar de la importancia del tiempo en la reconceptualización de los factores explicativos de la relación entre el entorno y la persona mayor, marcada por el curso de la vida, la religiosidad, la espiritualidad, las influencias históricas y las diferencias generacionales (Scheidt \& Norris-Baker, 2003); los modelos teóricos más recientes se caracterizan por la falta de atención a la dimensión temporal (pasado, presente y futuro) en la explicación del comportamiento del adulto mayor en relación al entorno. Esta ausencia

\footnotetext{
14 El modelo ecológico de la competencia (Lawton \& Nahemow, 1973) formula que la conducta es una función de la competencia del individuo (salud física, capacidades sensoriales y de percepción, motricidad y capacidad cognitiva) y de la presión ambiental (barreras arquitectónicas, violencia) a la que está expuesto. De lo que se deduce que el comportamiento del adulto mayor está determinado por la combinación del grado de presión ambiental y el nivel de competencia específico del sujeto. Así, el geógrafo Rowles (1978) expresó que las barreras ambientales se vuelven más significativas al enfrentarse con una disminución del estado fisiológico y de salud.

15 Powel Lawton (1999) reconoce la existencia de interacciones reciprocas, lo que se traduce en que las personas pueden actuar como agentes de los cambios ambientales, reconociéndose una relación bidireccional entre la persona mayor y el ambiente (hipótesis de la proactividad ambiental). Esta línea ha impulsado los estudios de gestión ambiental y confort ambiental del envejecimiento.
} 
Figura $\mathrm{N}^{0} 1$

Análisis de la experiencia socio-espacio-temporal de envejecer en el lugar

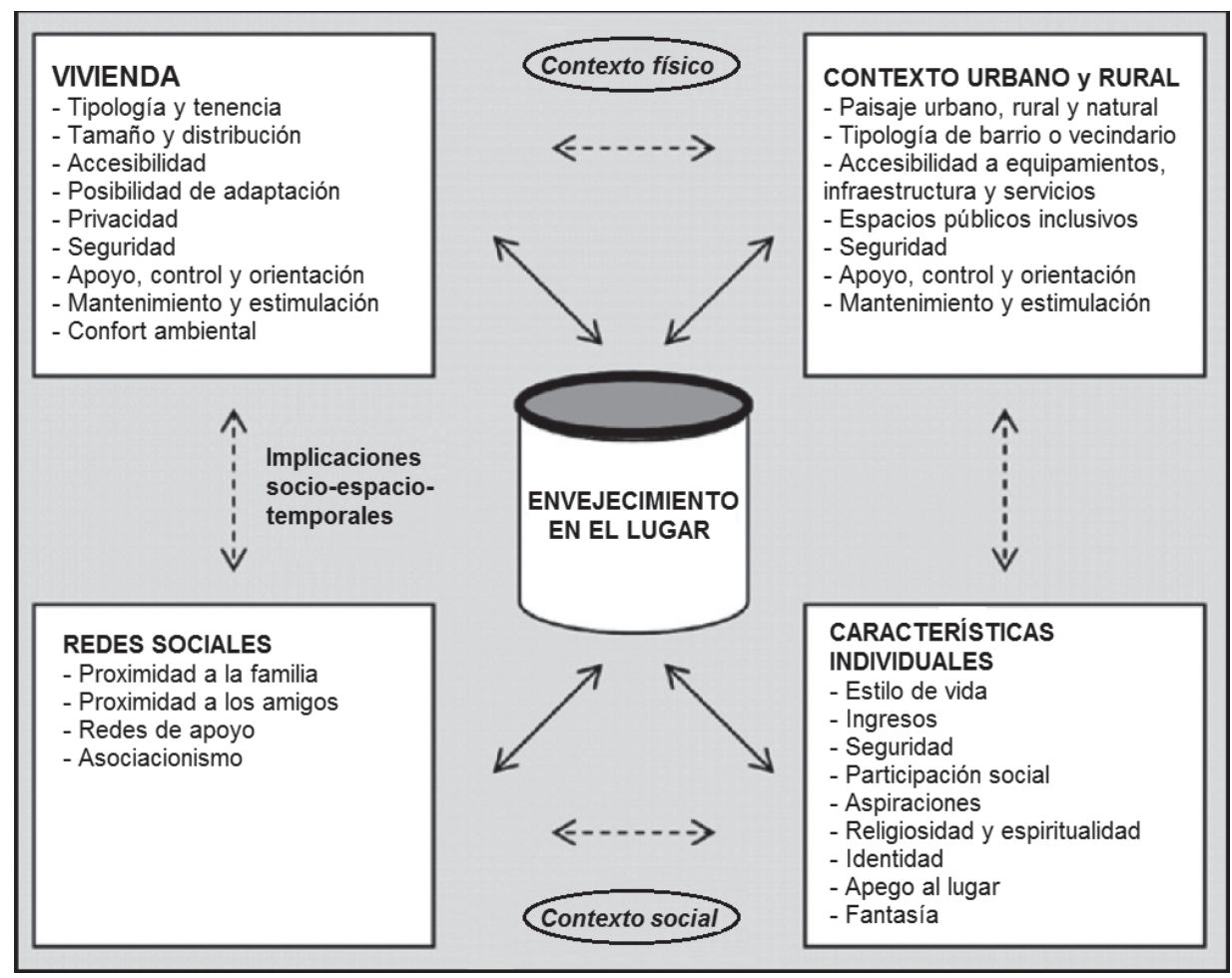

Fuente: Elaboración propia en base a Kahana et al. (2003) y Davies \& James (2011).

es achacable a la escasez de estudios longitudinales, lo que impide comprobar que a lo largo de la vida las experiencias espaciales (ambiente físico, cultural y social) determinan como se envejece en el entorno. De ahí, la necesidad de favorecer estudios longitudinales sobre la preferencia de envejecer en el lugar (sus viviendas, su barrios y su gente), incluso cuando se enfrentan a situaciones de riesgo y fragilidad $^{16}$. En la misma línea, se defiende la articulación de estrategias metodológicas para la comprensión del envejecimiento a escala urbana a través de la dimensión espacio-tiempo (Nóbrega, 2014).

\footnotetext{
${ }^{16}$ Se sugiere que las personas que están familiarizadas con su entorno cotidiano son más independientes y presentan un mayor nivel de competencia. Por ejemplo, se ha constatado que las personas mayores frágiles son capaces de permanecer independientes al beneficiarse de entornos a los que están acostumbrados (importancia del lugar) (Rowles, 1978).
}

A la fecha, debemos seguir desvelando los secretos del apego y la identidad en el deseo de envejecer en el lugar, incluso en contextos desfavorables (Smith, 2009). Una vía de investigación geográfica se centraría en el papel de la fantasía sobre el lugar, no solo como un recurso que favorece la adaptación al entorno (Rowles, 1978), sino como un posible factor de exclusión socioespacial. Igualmente, para desentrañar las interrogantes asociadas a los factores que moldean el carácter y la experiencia de envejecer en el lugar, proponemos el análisis de las implicaciones socio-espacio-temporales de la vivienda, el contexto urbano y rural, las relaciones sociales y las características individuales, en términos de distancia física y temporal (Figura $\left.N^{\circ} 1\right)$.

En los últimos años se están realizado importantes contribuciones en el estudio de los atributos y funciones del contexto ambiental (lugar) y su influencia en el ser 
humano (Carp \& Carp, 1984; Whahl, 2007; Kurniawati, 2012), sin embargo, ha faltado consenso en la definición y estratificación de las dimensiones objetivas y subjetivas del ambiente del adulto mayor (Wahl et al., 2012), circunstancia que se ha visto agudizada por el predominio de estudios descriptivos y escasamente analíticos en su abordaje. Al respecto, definimos los atributos como todo lo objetivo del ambiente que se encuentra fuera de la piel, que es inanimado, mesurable a partir de unidades del sistema métrico decimal (centímetros, gramos, segundos, grados), y que caracteriza al medio ambiente. A su vez, los atributos pueden ser medidos por su funcionalidad (calidad ambiental, proximidad y movilidad) y su usabilidad (áreas verdes, equipamientos, legibilidad). Asimismo, las funciones se refieren a todo lo subjetivo del ambiente vinculado a la gama de lazos afectivos y cognitivos que el adulto mayor construye con su entorno (lugar), es decir, las diferentes funciones del ambiente que pueden ser de utilidad para una persona que envejece. Dichas funciones pueden ser medidas por su funcionalidad (identidad, autonomía, familiaridad, agradabilidad e interacción social) y usabilidad (bienestar, seguridad, versatilidad, apego al lugar). Además, los atributos y funciones deben favorecer la comprensión del ambiente físico-social en el envejecimiento, asociado al acceso y vínculos, los usos y actividades, el confort e imagen, y la sociabilidad (Figura $\mathrm{N}^{\circ} 2$ ).

Figura $\mathrm{N}^{\circ} 2$

Atributos y funciones del lugar en el envejecimiento

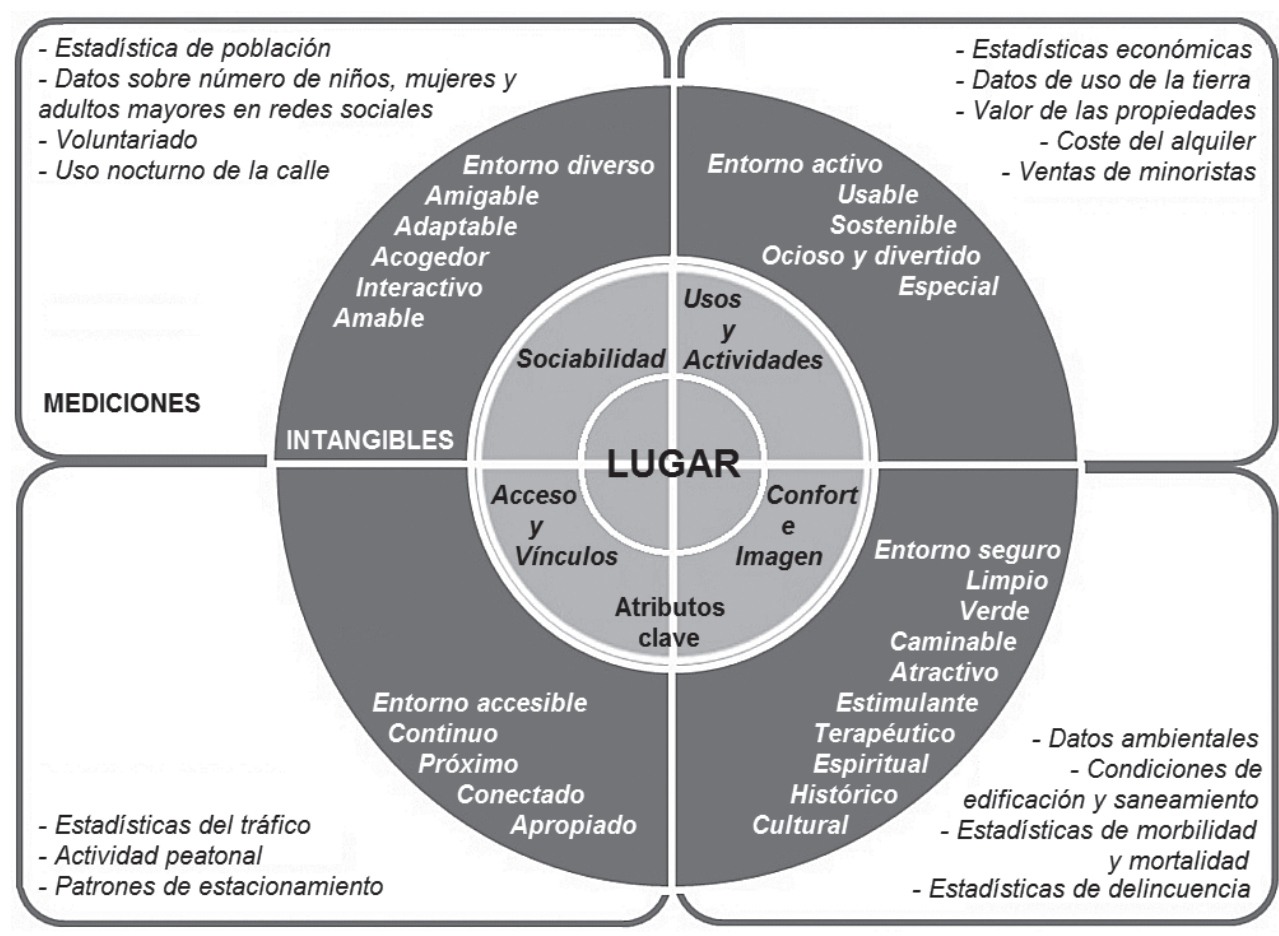

Fuente: Elaboración propia en base a trabajo de Kurniawati (2012).

En este sentido, consideramos que se debe desestimar la aplicación directa de modelos teóricos y metodológicos diseñados en otros contextos geográficos y culturales (países anglosajones), obligando a realizar adaptaciones de los modelos a partir de las 
características ambientales, culturales y psicosociales de los entornos físico-sociales del envejecimiento en la región. Como respuesta al problema, planteamos la necesidad de conceptualizar las principales dimensiones del entorno físico-social del envejecimiento, a través de identificar cuándo, dónde y para quién ciertos atributos y funciones ambientales son más determinantes en el envejecimiento en el lugar.

Entre los principales problemas metodológicos se subraya las imprecisas mediciones y asociaciones, a través de factores de confusión achacables a los problemas conceptuales, que han invalidado los supuestos hallazgos de determinadas investigaciones (Panter \& Jones, 2010). A ello habría contribuido las omisiones relativas a la justificación del método de medición empleado en la evaluación de los atributos y funciones del entorno físico-construido (Gebel et al., 2007), así como el impreciso uso de las escalas de análisis espaciales y temporales. También, sería criticable la utilización de fuentes primarias sin especificar el origen y vigencia de las mismas (marco espacial y temporal), y los posibles sesgos en la selección de la muestra (tipologías de sujetos y ambientes) (Heath et al., 2006; Sánchez-González, 2008). Por ejemplo, se han registrado dudosas asociaciones entre atributos de ambientes de recreación (paisajes urbanos, espacios públicos) y el medio de transporte empleado por el usuario adulto mayor (Schaie \& Pietrucha, 2000; Saelens \& Handy, 2008). En la misma línea, advertimos que las metodologías empleadas en los estudios de distribución y localización del envejecimiento han sido insuficientes para determinar áreas homogéneas de la población anciana con similares características sociodemográficas y ambientales, lo que ha redundado en visiones sesgadas de este colectivo y sus entornos.

En los últimos años algunos investigadores (Schwarz, 2012) plantean una gerontología ambiental focalizada principalmente en la utilización de métodos cualitativos, haciendo una dura crítica a la etapa positivista que predominó en las décadas pasadas. Sin embargo, consideramos que, si bien la crítica está razonada, no compartimos su valoración general hacia ambos métodos de análisis. Por una parte, los métodos cualitativos posibilitan una valiosa aproximación situacional a la realidad. A pesar ello, en diferentes investigaciones cualitativas se observa una escasa replicación de estudios para validar las categorías y estandarizar los intervalos, restando confianza a las escalas de análisis del ambiente físico-construido del envejecimiento. Por otra parte, criticamos el supuesto divorcio entre ambos métodos en la comprensión del fenómeno, más bien, nos posicionamos a favor del avance de la gerontología ambiental y, especialmente de la geografía, a partir de la combinación de los métodos cualitativos y cuantitativos. Así, recientes estudios geográficos (Garvin et al., 2012) emplean distintos métodos cualitativos, como la foto-provocación ${ }^{17}$ y las representaciones en diseño ambiental ${ }^{18}$. Por su parte, entre los métodos cuantitativos se emplean análisis geoestadísticos multivariados mediante el uso de Sistemas de Posicionamiento Global (GPS), Tecnologías de Identificación por Radiofrecuencia (RFID), sensores ambientales y la realidad virtual, con objeto de construir un sistema de monitoreo en el lugar (interior de la vivienda, espacio urbano) (Hanson, 2004; Lin et al., 2006; Kaczynski \& Henderson, 2008); así como de Sistemas de Información Geográfica (SIG), por ejemplo, en el estudio de la distribución de los servicios de salud en relación a la vulnerabilidad socioespacial de las personas ancianas según barrios (SánchezGonzález, 2009b). Al respecto, se subraya la importancia de hacer un uso más racional de los SIG (Hawthorne \& Kwan, 2012), a través de implicaciones teóricas asociadas a la reconceptualización de la accesibilidad (potencial y real) de los adultos mayores a los servicios sociales y de salud, abogando por una distribución equitativa, a partir de la planifi-

\footnotetext{
17 La fotoprovocación es un método etnográfico, consistente en que los investigadores introducen fotografías, tanto del entrevistado como del investigador, en el contexto de la entrevista para ampliar las preguntas y la información proporcionada a través de la imagen comentada (Clark, 2004).

18 Las representaciones en diseño ambiental son un método etnográfico basado el empleo de las técnicas de representación y expresión artística de las humanidades y las artes, a través de la acción participativa de la comunidad, con el propósito de propiciar experiencias vivenciales asociadas a las visitas de campo, con objeto de evocar representaciones de los ambientes de la vida diaria y favorecer propuestas sobre diseño ambiental (Boeck et al., 2011).
} 
cación urbana gerontológica, que incorpore aspectos como la calidad de la atención y la satisfacción del usuario de avanzada edad.

\section{Discusión y conclusiones}

Los recientes avances de la gerontología ambiental confirman que sus contribuciones, especialmente desde la geografía, están permitiendo responder a las importantes interrogantes sobre los ambientes físico-sociales y sus implicaciones en la calidad de vida de las personas mayores. Dichas aportaciones multidisciplinares teóricas y metodológicas, no exentas de crítica, están posibilitando la comprensión del envejecimiento saludable en el lugar a través de su relación con los ambientes físico-construidos seguros, proactivos, terapéuticos, estimulantes y simbólicos, que potencian las actividades saludables de la vida cotidiana y el apego e identidad de lugar; y con los entornos sociales, que fomentan las relaciones sociales, la participación e integración social, así como facilitan la prestación e intercambio de ayuda. Así, es necesario un abordaje interdisciplinar del análisis del ambiente tanto físico como social, evitando el estudio parcial de esas dimensiones de forma aislada y desconectada, que no permiten una visión integral de la compleja relación entre los ambientes (físico-sociales) urbanos y rurales, y la calidad de vida de las personas mayores.

En América Latina la escasa introducción de la gerontología ambiental y de la geografía del envejecimiento está motivada, en parte, por el dominio de literatura anglosajona basada en el empleo de conceptos, así como de modelos teóricos y metodológicos, en muchas ocasiones, alejados de la realidad de los contextos latinos. De lo cual deducimos que el limitado avance teórico y metodológico en el análisis del ambiente físico-construido del envejecimiento, en parte, se debería a la mayor tradición de los estudios del entorno social del envejecimiento entre determinados profesionales de las ciencias sociales y de la salud (trabajadores sociales, psicólogos), así como la escasa presencia de otros profesionales (arquitectos, geógrafos, urbanistas). Todo ello explica la reiterada inconsistencia en la definición del concepto de lugar en el envejecimiento, ya sea asociado a un barrio residencial o un lugar de trabajo. De hecho, en la producción científica latinoamericana se observa un limitado abordaje geográfico de la cuestión ambiental del envejecimiento desde una perspectiva gerontológica, así como un desigual impacto del tema en la región. Al respecto, el fenómeno ha sido abordado de forma desigual en los países de la región, destacando Brasil y México. Esta circunstancia no obedece tanto al avance del proceso del envejecimiento demográfico a nivel nacional, como al desarrollo de la investigación geográfica de esos países, así como al interés de distintos investigadores de las ciencias sociales y de la salud por las implicaciones socioespaciales del fenómeno. También, el dominio del inglés como ve hículo de la comunicación científica (revistas geográficas y gerontológicas), minimiza el impacto de las investigaciones realizadas en América Latina y publicadas en revistas de otros idiomas (español, portugués) (Bajerski, 2011). Igualmente, no se han encontrado manuales en castellano o portugués que faciliten su comprensión por profesionales, gestores, y público en general. También, el escaso impacto de la investigación regional sobre el envejecimiento en el lugar ha estado condicionado por la falta de iniciativa pública y privada, la limitada comunicación entre los investigadores, y el inmovilismo académico en las universidades. Por ello, planteamos el desafío de iniciar una labor pedagógica entre académicos y responsables públicos sobre la importancia del tema, con objeto de favorecer los esfuerzos necesarios para desarrollar estudios longitudinales transnacionales sobre el envejecimiento en el lugar, con objeto de propiciar resultados extrapolables a partir de compartir bases de datos y metodologías comunes. Asimismo, demandamos una mayor participación activa de las personas mayores en la planificación de sus entornos cotidianos en la región; para lo cual es necesario diseñar herramientas para analizar los atributos y funciones del entorno físico-social. También, dichas herramientas deben facilitar la evaluación de los ambientes urbanos y rurales, tanto a profesionales y gestores, como a los propios adultos mayores y sus familias.

Estamos empezando a desentrañar los enigmas asociados a las relaciones entre el ambiente y el envejecimiento, ya que, se está descubriendo que la forma de envejecer depende en buena medida del lugar donde se 
vive y de la estimulación psicosocial que recibe. De ahí, la necesidad de contribuir a favorecer una mejor identificación y definición del concepto de lugar (vivienda, barrio, espacios públicos, lugar de trabajo), desarrollando análisis más detallados de las dimensiones y variables específicas del entorno construido (propiedades relativas a la calidad, accesibilidad, usabilidad), a través del uso racional de las herramientas (SIG) y la combinación de variados métodos cualitativos (fotoprovocación) y cuantitativos (sensores, realidad virtual). En este sentido, se deben incorporar medidas objetivas y subjetivas del ambiente construido, excluyendo factores de confusión que redundan en explicaciones alternativas poco concluyentes. Del mismo modo, es fundamental una mayor clarificación metodológica y la incorporación de estudios longitudinales, que están contribuyendo a la comprensión de procesos complejos, como la movilidad residencial en la jubilación. Para ello, en América Latina es necesaria una mayor implicación de los investigadores de las ciencias sociales, en especial de los geógrafos, sobre las cuestiones ambientales de la gerontología, a través de la formación y especialización. De igual forma, se defiende la conveniencia de implementar el conocimiento teórico y metodológico geográfico en gerontología, a través del enfoque ecológico, la definición del concepto de medio ambiente y el análisis geoestadístico mediante SIG.

Hoy sabemos, a través de los estudios geográficos, que la curiosidad y el comportamiento exploratorio disminuye con la edad, lo que favorece una disminución de los patrones de movilidad y una mayor regularidad de las actividades de la vida cotidiana fuera del hogar (escala barrio). Esta circunstancia abre enormes posibilidades de investigación para impulsar la construcción de ambientes seguros, estimulantes y terapéuticos que despierten la curiosidad y favorezcan el envejecimiento activo.

Hemos aprendido que existe una estrecha relación entre el entorno físico-social y el estado de salud en la vejez, como una etapa vital y dinámica en la que se producen cambios, traducidos en ganancias y pérdidas, que no tienen el mismo significado para las distintas cohortes de adultos mayores. Del mismo modo, la capacidad funcional frente a diferentes ambientes construidos (vivienda, barrio, hospitales) varía de forma significativa con la edad, influyendo en la autonomía e independencia de las personas de edad avanzada. Por lo tanto, en el análisis del envejecimiento en el lugar se recomienda realizar una adecuada estratificación de la población según edad y género, a partir de las diferencias geográficas, socioeconómicas y culturales de los heterogéneos colectivos sociales (inmigrantes, indígenas, discapacitados). Precisamente, será necesario desarrollar nuevos estudios longitudinales basados en el análisis de las implicaciones socio-espacio-temporales del envejecimiento en el lugar a partir de la definición y clasificación de los atributos y funciones del entorno de la vivienda, el contexto urbano, las relaciones sociales y las características individuales del adulto mayor.

En los próximos años las políticas de salud asociadas al envejecimiento de la población deben priorizar estrategias de prevención de enfermedades crónicas (problemas cardiorrespiratorios, diabetes, obesidad), psicosociales (depresión, ansiedad) y neurodegenerativas (alzhéimer, párkinson), a través de actuaciones en el contexto físico-social (viviendas, residencias, hospitales, espacios urbanos), lo que se traducirá en una mejor gestión del gasto farmacéutico y hospitalario. Asimismo, en la región la planificación gerontológica deberá implicar soluciones integrales para los adultos mayores y sus familias, basadas en la investigación de los entornos, así como en el uso racional de los recursos desde necesarios ejercicios de calidad y eficiencia, basados en los principios éticos de equidad y justicia distributiva.

En el ámbito de la investigación de la gerontología ambiental y la geografía del envejecimiento, necesitamos favorecer puntos de encuentro a partir de la discusión y la búsqueda de consensos frente a la dispersión de modelos teóricos y metodológicos. Es ineludible que en las futuras investigaciones sobre el entorno del envejecimiento se adopten metodologías de evaluación más sistemáticas para ayudar en la síntesis de la evidencia. Precisamente, en el siglo XXI en la región los retos del envejecimiento demográfico asociados al cambio climático deben impulsar nuevos estudios, conformados por equipos multidisciplinares y abordajes interdisciplinares, sobre las implicaciones del 
urbanismo formal e informal en el agravamiento de los efectos del clima sobre la salud de la población envejecida. Además, las nuevas investigaciones sobre las características objetivas y percibidas del vecindario contribuirán al desarrollo de la planificación urbana gerontológica, a través de posibilitar intervenciones amigables con este colectivo en las ciudades de América Latina

Como todo campo multidisciplinar que está en ciernes, la gerontología ambiental presenta lagunas y críticas a sus modelos en construcción, pero estamos convencidos de su importancia futura a nivel internacional y, sobre todo, en la región, así como la necesidad de edificar puentes teóricos y metodológicos con la ayuda de la geografía. Por ello, no debemos olvidar que las soluciones a esta cuestión central del envejecimiento y su entorno, vendrá no solo a través del avance científico (entornos inteligentes, nuevas propuestas de movilidad y productos tecnológicos), sino, sobre todo, de la toma de conciencia sobre el fenómeno del envejecimiento y la importancia de construir entornos amigables que refuercen nuestros lasos afectivos con la comunidad.

\section{Referencias bibliográficas}

ANDREWS, G.J.; CUTCHINB, M.; MCCRACKENC, K.; PHILLIPS, D.R.; \& WILESE, J. Geographical Gerontology: The constitution of a discipline. Social Science \& Medicine, 2007, Vol. 65, № 1, p. 151-168.

ANDREWS, G.J. \& PHILLIPS, D.R. Ageing and Place: Perspectives, Policy, Practice. London: Routledge, 2005.

BAGNULO, C. y PIZARRO, N. Distribución espacial de los adultos mayores en condición de riesgo de malnutrición en la ciudad de Bahía Blanca. Revista Universitaria de Geografía, 2010, Vol. 19, № 1, p. 119-138.

BAJERSKI, A. The role of French, German and Spanish journals in scientific communication in international geography. Area, 2011, Vol. 43, No3, p. 305-313.

BALSAN, R. Espaços de turismo e lazer dos idosos em Rio Claro-Sp. Rio Claro-Sp:
Tese doutorado, Universidade Estadual Paulista, 2005.

BALSAN, R. Atividades de lazer dos idosos na cidade: um estudo de caso na cidade de Rio Claro-Sp. Geografando, 2006, Vol. 1, $N^{\circ} 2$, p. 65-87.

BELLO, W. Vulnerabilidad socio-demográfica de las personas de la tercera edad. Estudio de caso, centro histórico de La Habana. Cuadernos Geográficos, 2013, № 52, p. 153-177.

BOECK, D.; MOXLEY, D. \& WACHTER, H. The Infusion of the Arts and Humanities into a Community Needs Assessment of Aging. Rationale Guiding the Environmental Design Exhibit in Norman, Oklahoma. Proceedings Submittal for the 9th Annual Hawaii International Conference on Arts and Humanities, Honolulu, Hawaii, 2011, p. 1-13.

CAO, X.J. Exploring causal effects of neighborhood type on walking behavior using stratification on the propensity score. Environment and Planning A, 2010, Vol. 42, № 2, p. 487-504.

CAPRON, G. y GONZÁLEZ, S. Movilidad residencial de los adultos mayores y trayectorias de vida familiares en la ZMVM. Alteridades, 2010, Vol. 20, No 39, p. 67-78.

CARP, F.M. \& CARP, A. A complementary/ congruence model of well-being or mental health for the community elderly. Elderly People and the Environment Human Behavior and Environment, 1984, Vol. 7, p. 279-336.

CEI. Os idosos do Rio Grande do Sul: estudo multidimensional de suas condições de vida. Porto Alegre: Conselho Estadual do Idoso, 1997.

CHACKIEL, J. El envejecimiento de la población latinoamericana: ¿hacia una relación de dependencia favorable? Santiago de Chile: Comisión Económica para América Latina y el Caribe, 2000.

CHÁVEZ, R. y SÁNCHEZ-GONZÁLEZ, D. Vulnerabilidad social de los adultos mayores retornados en la frontera de México y Estados Unidos. En: EGEA, C.; SÁNCHEZ-GONZÁ- 
LEZ, D.; y SOLEDAD, J.I. (coordinadores). Vulnerabilidad social: posicionamiento y ángulos desde geografías diferentes. Granada: Universidad de Granada, 2012, p. 223-240.

CLARK, M. Framing the Social World With Photo-Elicitation Interviews. American Behavioral Scientist August, 2004, № 47, p. 1507-1527.

DAVIES, A. \& JAMES, A. Geographies of Ageing. Social Processes and the Spatial Unevenness of Population Ageing. Burlington: Ashgate Publishing Company, 2011.

DÍAZ-TENDERO, A. Estudios de población y enfoques de gerontología social en México. Papeles de Población, 2011, Vol. 17, No 70, p. 49-79.

DUARTE, F.; FERNANDES, F.M. \& NICOLAU, G.K. Experiências Cartográficas com Pessoas Idosas: Uma busca por Novos Olhares e Leituras Sociais. Holos, 2009, Vol. 25, $N^{\circ} 3$, p. 63-72.

ESCUDERO, J.M. Los viejos en su casa, en su ciudad. Scripta Nova. Revista Electrónica de Geografía y Ciencias Sociales, 2003, Vol. VII, No 146 (203). Disponible en Internet: http://www.ub.es/geocrit/sn/sn-146(103).htm

FADDA, G. y CORTÉS, A. Hábitat y adulto mayor: el caso de Valparaíso. Revista INVI, 2009, Vol. 24, No 66, p. 89-113.

FATIGA, B.C. Lugar e o não-lugar de idosos institucionalizados: o idoso e a tal da Geografia. Revista Portal de Divulgação, 2012, Vol. 2, No 23, p. 8-16.

FERNÁNDEZ-TROCÓNIZ, M.I. y FERNÁNDEZ-BALLESTEROS, R. Modelos ambientales sobre la vejez. Anales de psicología, 1990, Vol. 6, No 2, p. 181-198.

FETTER, I.; OLIVOS, L.; GUTIÉRREZ, G.; y MICHÁN, S. Regulación Epigenética del envejecimiento. En: LÓPEZ MUÑOZ, E. y TORRES, N.M. (coordinadores). Aspectos moleculares del envejecimiento. México: Instituto de Geriatría, 2012, p. 61-69.

FORMIGA, N. y PRIETO, MB. Envejecimiento y diferenciación socioespacial en calidad de vida. La situación en Bahía Blanca, Argentina. En: PELÁEZ, E. (coordinador). Sociedad y adulto mayor en América Latina. Estudios sobre Envejecimiento en la Región. Córdoba: Editorial Copiar-ALAP, 2010, p. 3967.

GARVIN, T.; NYKIFORUK, C.I.J. \& JOHNSON, S. Can we get old here? Seniors' perceptions of seasonal constraints of neighbourhood built environments in a northern, winter city. Geografiska Annaler: Series B, Human Geography, 2012, № 94, p. 369-389.

GASTALDO, D.; ANDREWS, G.J. \& KHANLOU, N. Therapeutic landscapes of the mind: Theorizing some intersections of health geography, health promotion and immigration studies. Critical Public Health, 2004, Vol. 14, № 2, p. 157-176.

GEBEL, K.; BAUMAN, A.E. \& PETTICREW, $M$. The physical environment and physical activity: a critical appraisal of review articles. American Journal of Preventive Medicine, 2007, Vol. 32, No 5, p. 361-369.

GUIDOTTI, C.A. y AIDAR, T. Condiciones de vida del adulto mayor en el ámbito doméstico: envejecimiento y transferencias en Montevideo-Uruguay. Papeles de Población, 2012, Vol. 18, № 74, p. 171-202.

GOLANT, S.M. The quest for residential normalcy by older adults: Relocation but one pathway. Journal of Aging Studies, 2011, Vol. 25, № 3, p. 193-205.

GOLANT, S.M. \& SALMON, J.R. The unequal availability of affordable assisted living units in Florida's Counties. Journal of Applied Gerontology, 2004, Vol. 23, № 4, p. 349-369.

GOMES DA CONCEIQÁO, M.C. El envejecimiento poblacional y las formas de residencia en México. Papeles de Población, 1997, Vol. 3, No 14, p. 171-194.

GÓMEZ, J.F. y CURCIO, C.L. Envejecimiento rural: el anciano en las zonas cafeteras colombianas. Manizales: Universidad de Caldas, 2004.

GUTIÉRREZ, L.M. México y la revolución de la longevidad. En: GUTIÉRREZ, L.M. y GU- 
TIÉRREZ-ÁVILA, J.H. (coordinadores). Envejecimiento humano. Una visión transdisciplinaria. México: Instituto de Geriatría, 2010, p. 21-36.

GUZMÁN, J.M. Envejecimiento y desarrollo en América Latina y el Caribe. Santiago de Chile: Comisión Económica para América Latina y el Caribe, 2002.

HAKKERT, R. y GUZMÁN, J.M. Envejecimiento demográfico y arreglos familiares de vida en América Latina. En: ARIZA, M. y OLIVE, O. (coordinadores). Imágenes de la familia en el cambio de siglo. México: Universidad Nacional Autónoma de México, 2004, p. 479-518.

HAM-CHADE, R.; PALLONI, A. \& WONG, R. Aging in developing countries: Building bridges for integrated research agendas. Paris: IUSSP, 2009

HANSON, T.R. Using GPS and GIS to study the travel habits of elderly drivers. Fredericton: University of New Brunswick, 2004.

HARPER, S. \& LAWS, G. Rethinking the geography of ageing. Progress in Human Geography, 1995, Vol. 19, No 2, p. 199-221.

HAWTHORNE, T.L. \& KWAN, M.P. Using GIS and perceived distance to understand the unequal geographies of healthcare in lower-income urban neighbourhoods. The Geographical Journal, 2012, Vol. 178, № 1, p. 18-30.

HAYES, M.F. Una nueva migración económica: el arbitraje geográfico de los jubilados estadounidenses hacia los países andinos. Boletín del Sistema de Información sobre Migraciones Andinas, 2013, № 15, p. 2-13.

HEATH, G.W.; BROWNSON, R.C.; KRUGER, J.; MILES, R.; POWELL, K.E. \& RAMSEY, L.T. The effectiveness of urban design and land use and transport policies and practices to increase physical activity: a systematic review. Journal of Physical Activity and Health, 2006, Vol. 3, No S1, p. S55-S76.

HUENCHUAN, S. Envejecimiento, derechos humanos y políticas públicas. Santiago de Chile: Comisión Económica para América Latina y el Caribe, 2009.
JACOBSON, K. Embodied Domestics, Embodied Politics: Women, Home, and Agoraphobia. Human Studies, 2011, Vol. 34, No 1, p. 1-21.

JACOMINI, W. O envelhecimento da população e a condição dos idosos: Processos sociais analisados em Rio Claro. Rio Claro-Sp: Universidade Estadual Paulista, 1990.

JASSO, P.B.; MONTOYA, J.; y CADENA, E. Los adultos mayores en las zonas metropolitanas de México: desigualdad socioeconómica y distribución espacial, 1990-2005. Papeles de Población, 2011, Vol. 17, No 70, p. 81124.

JORGE, A.C. Os idosos e a circulação no espaço urbano: a locomoção dos idosos do Pólo Tuna Luso Brasileira do Projeto Vida Ativa na cidade de Belém/PA. Belem: Dissertação, Universidade da Amazônia, 2009.

KACZYNSKI, A.T. \& HENDERSON, K.A. Parks and recreation settings and active living: a review of associations with physical activity function and intensity. Journal of Physical Activity and Health, 2008, Vol. 5, No 4, p. 619-632.

KAHANA, E.; LOVEGREEN, L.; KAHANA, B. \& KAHANA, M. Person, environment, and person-environment fit as influences on residential satisfaction of elders. Environment and Behavior, 2003, Vol. 35, № 3, p. 434-453.

KIY, R. \& McENANY, A. Housing and Real Estate Trends among Americans Retiring in Mexico's Coastal Communities. U.S. Retirement in Mexico Research Series, 2010, No 3, p. 1-33.

KURNIAWATI, W. Public Space for Marginal People. Procedia. Social and Behavioral Sciences, 2012, Vol. 36, p. 476-484.

LAWTON, M.P. Environmental taxonomy: generalizations from research with older adults. In: FRIEDMAN, S.L. \& WACHS, T.D. (editors). Measuring Environment across the Life Span. Washington: American Psychological Association, 1999, p. 91-124.

LAWTON, M.P. An environmental psychologist ages. In: ALTMAN, I. \& CHRISTENSE, K. 
(editors). Environmental and behavior studies: Emergence of intellectual traditions. New York: Plenum Press, 1990, p. 339-363.

LAWTON, M.P. The elderly in context: Perspectives from environmental psychology and gerontology. Environment and Behaviour, 1985, Vol. 17, № 4, p. 501-519.

LAWTON, M.P. \& NAHEMOW, L. Ecology and the aging process. In: EISDORFER, C. \& LAWTON, M.P. (editors). The psychology of adult development and aging. Washington DC: American Psychological Association, 1973, p. 619-674.

LIN, C.; CHIU, M.; HSIAO, C. \& LEE, R. Wireless Health Care Service System for Elderly With Dementia. Information Technology in Biomedicine, 2006, Vol. 10, № 4, p. 696-704.

LIZÁRRAGA, O. La inmigración de jubilados estadounidenses en México y sus prácticas transnacionales: Estudio de caso en Mazatlán, Sinaloa y Cabo San Lucas, Baja California Sur. Migración y Desarrollo, 2008, No 11, p. 97-117.

LÓPEZ-SALGADO, M.N. Políticas de viviendas para nuevas demandas: el caso del programa de jubilados y pensionistas del BPSMVOTMA del Uruguay. Revista INVI, 2006, Vol. 21, No 57, p. 44-60.

MATHIAS, T.A.F.; JORGE, M.H. \& ANDRADE, O.G. Morbimortalidade por causas externas na população idosa residente em município da região sul do Brasil. Revista Latino-Americana de Enfermagem, 2006, Vol. $14, N^{\circ} 1$, p. 17-24.

MEJÍA, S.; JAIMES, A.M.; VILLA, A.; RUIZ, L.; y GUTIÉRREZ, L.M. Deterioro cognoscitivo y factores asociados en adultos mayores en México. Salud pública de México, 2007, Vol. 49, № Extra 4, p. 475-481.

NARVÁEZ, O. Envejecimiento demográfico y requerimientos de equipamiento urbano. Papeles de Población, 2012, Vol. 18, № 74, p. 203-235.

NEGRETE, M.E. El envejecimiento poblacional en la ciudad de México: evolución y pautas de distribución espacial entre 1970-
2000. Papeles de Población, 2003, Vol. 9, № 37, p. 5-27.

NÓBREGA, P.R.C. Notas sobre a relação sociedade e a natureza e a emergência do envelhecimento como tema complexo: Um olhar geográfico. Geografia em Questão, 2014, Vol. 7, № 1, p. 87-103.

ORGANIZACIÓN MUNDIAL DE LA SALUD (OMS). Ciudades globales amigables con los mayores: Una guía. Ginebra: Organización Mundial de la Salud, 2007.

ORGANIZACIÓN DE LAS NACIONES UNIDAS (ONU). World Population Ageing 2013. Report. New York: Department of Economic and Social Affairs Population Division, United Nations, 2013.

PANTER, J.R. \& JONES, A. Attitudes and the environment as determinants of active travel in adults: what do and don't we know? Journal of Physical Activity Health, 2010, Vol. 7, № 4, p. 551-561.

PARRA, D.C.; GOMEZ, L.F.; SARMIENTO, O.L.; BUCHNERE, D.; BROWNSONA, R.; SCHIMD, T.; GOMEZ, V. \& LOBELO, F. Perceived and objective neighborhood environment attributes and health related quality of life among the elderly in Bogotá, Colombia. Social Science \& Medicine, 2010, Vol. 70, № 7, p. 1070-1076.

PEACE, S.; HOLLAND, C. \& KELLAHER, L. Environment and Identity in Later Life. Growing Older. Maidenhead: Open University Press, 2006.

PEIXOTO, A.; RAMOS, E.; ATIE, S.; CRISPIM, A. \& ORLANDO, A. A influência das quedas na qualidade de vida de idosos. Ciência \& Saúde Coletiva, 2008, Vol. 13, № 4, p. 1265-1273.

PÉRICLES, E.; SIQUEIRA, R. \& FLORINDO, AA. A prática de caminhada como forma de deslocamento e sua associação com a percepção do ambiente em idosos. Revista Brasileira de Atividade Física \& Saúde, 2009, Vol. 14, № 3, p. 197-205.

PIZARRO, N. El problema de la mortalidad y la geografía de la ancianidad en Bahía 
Blanca. Bahía Blanca: Tesis doctoral, Universidad Nacional del Sur, 2005.

ROSENBERG, M.W. Medical or Health Geography? Populations, Peoples and Places. International Journal of Population Geography, 1998, Vol. 4, No 3, p. 211-226.

ROWLES, G.D. Prisoners of space? Exploring the geographical experience of older people. Boulder: Westview Press, 1978.

ROWLES, G.D. \& BERNARD, M. Environmental Gerontology: Making Meaningful Places in Old Age. New York: Springer Publishing Company, 2013.

ROWLES, G.D. \& CHAUDHURY, H. Home and identity in late life: international perspectives. New York: Springer Publishing Company, 2005.

SAELENS, B.E. \& HANDY, S.L. Built environment correlates of walking: a review. Medicine and Science in Sports and Exercise, 2008, Vol. 40, No 7 S, p. 550-566.

SALAS, S.M. y SÁNCHEZ-GONZÁLEZ, D. Envejecimiento de la población, salud y ambiente urbano en América Latina. Retos del Urbanismo Gerontológico. Contexto. Revista de la Facultad de Arquitectura de la Universidad Autónoma de Nuevo León, 2014, Vol. 8, $\mathrm{N}^{\circ}$ 9, p. 31-49.

SALDIVA, P.H.N.; POPE, C.A.; SCHWARTZ, J.; DOCKERY, D.W.; LICHTENFELS, A.J.; SALGE, J.M.; BARONE, I. \& MIKLOS, G. Air Pollution and Mortality in Elderly People: A TimeSeries Study in Sao Paulo, Brazil. Archives of Environmental Health: An International Journal, 1995, Vol. 50, № 2, p. 159-163.

SÁNCHEZ-GONZÁLEZ, D. Envejecimiento demográfico urbano y sus repercusiones socioespaciales en México: Retos de la Planeación Gerontológica. Revista de Geografía Norte Grande, 2007, № 38, p. 45-61.

SÁNCHEZ-GONZÁLEZ, D. Adultos mayores en la planeación del espacio turístico rural en Tamaulipas. Papeles de Población, 2008, Vol. 14, No 55, p. 59-94.
SÁNCHEZ-GONZÁLEZ, D. Contexto ambiental y experiencia espacial de envejecer en el lugar: el caso de Granada. Papeles de Población, 2009a, Vol. 15, № 60, p. 175-213.

SÁNCHEZ-GONZÁLEZ, D. Geografía del envejecimiento vulnerable y su contexto ambiental en la ciudad de Granada: Discapacidad, dependencia y exclusión social. Cuadernos Geográficos, 2009b, Vol. 45, p. 107-135.

SÁNCHEZ-GONZÁLEZ, D. Geografía del envejecimiento y sus implicaciones en Gerontología. Contribuciones geográficas a la Gerontología Ambiental y el envejecimiento de la población. Saarbrücken: Editorial Académica Española, 2011.

SÁNCHEZ-GONZÁLEZ, D. y EGEA, C. Enfoque de vulnerabilidad social para investigar las desventajas socioambientales. Su aplicación en el estudio de los adultos mayores. Papeles de Población, 2011, Vol. 17, № 69, p. 151-185.

SÁNCHEZ-GONZÁLEZ, D. Prisioneros del espacio urbano. Retos de planificar ciudades amigables para las personas adultas mayores. En: PALACIOS, A.; SOUSA, E. y CADENA, E. (coordinadores). Espacio urbano, reconstrucción y reconfiguración territorial. Hualpén: Universidad del Bío-Bío, 2013, p. 101-118.

SÁNCHEZ-GONZÁLEZ, D. Identidad del lugar, envejecimiento y presiones ambientales de la ciudad. Reflexiones desde la gerontología ambiental. En: SÁNCHEZ-GONZÁLEZ, D. y DOMÍNGUEZ, L.A. (coordinadores). Identidad y espacio público. Ampliando ámbitos y prácticas. Barcelona: Editorial Gedisa, 2014, p. 141-168.

SCHAIE, K.W. \& PIETRUCHA, M. (editors). Mobility and Transportation in the Elderly. New York: Springer Publishing Company, 2000.

SCHEIDT, R.J. \& NORRIS-BAKER, C. The General Ecological Model. Revisited. Evolution, Current Status and Continuing ChaIlenges. In: WAHL, H.W.; SCHEIDT, R.J. \& WINDLEY, P.G. (editors). Annual Review of Gerontology and Geriatrics. Focus On Aging in context. Socio Physical Environments. New 
York: Springer Publishing Company, 2003, p. 34-58.

SCHWARZ, B. Environmental Gerontology: What now? Journal of Housing for the Elderly, 2012, Vol. 26, № 1-3, p. 4-19.

SERRANO, A.T.; ORTIZ, M.I. y VIDAL, R. La discapacidad en población geriátrica del Distrito Federal, México, año 2000. Un caso de la geografía de la población. Terra Nueva Etapa, 2009, Vol. 25, No 38, p. 15-35.

SHIGEMATSU, R.; SALLIS, J.F.; CONWAY, T.L.; SAELENS, B.E.; FRANK, L.D.; CAIN, K.; CHAPMAN, J.E. \& KING, A.C. Age differences in the relation of perceived neighbourhood environment to walking. Medicine \& Science in Sports \& Exercise, 2009, Vol. 41, $\mathrm{N}^{\circ} 2$, p. 314-321.

SILVEIRA, A. Meio ambiente e envelhecimento: desafio e alternativas para a sociedade brasileira. Uma abordagem ecológica. Lumen, 2003, Vol. 9, № 20, p. 75-93.

SMITH, A. Urban ageing. En Ageing in Urban Neighbourhoods. Place attachments and social exclusion. Bristol, UK: The Policy Press, 2009.

STOKOLS, D. The paradox of environmental Psychology. American Psychologist, 1995, No 50 , p. 821-837.

SYLVESTRE, G. The geography of aging: a geographical contribution to gerontology. In: KOSTER, R. (editor). Prairie Perspectives: Geographical Essays. Saskatoon: University of Winnipeg, Vol. 2, 1999, p. 214-224.

TAPIA, C.; VARELA, H.; BARRA, L.; UBILLA, M.D.; ITURRA, V.; COLLAO, C. y SILVA, R. Valoración multidimensional del envejecimiento en la ciudad de Antofagasta. Revista Médica de Chile, 2010, Vol. 138, No 4, p. 444-451.
TOMASINI, S.L.V. Envelhecimento e planejamento do ambiente construído: em busca de um enfoque interdisciplinar. Revista Brasileira de Ciências do Envelhecimento Humano, 2005, Vol. 2, No 1, p. 76-88.

TODOROVA, I.; GUZZARDO, MT; ADAMS, W.E. \& FALCÓN, LM. Gratitude and longing: Meanings of health in aging for Puerto Rican adults in the mainland. Journal of Health Psychology, 2014, Vol. 19, № 2, p. $1-12$.

TRUJILLO, Z.; BECERRA-PINO, M. y RIVAS-VERA, S. Latinoamérica envejece: visión geriátrica y gerontológica. México: McGrawHill Interamericana, 2007.

WARNES, A.M. Geographical questions in gerontology: needed directions for research. Progress in Human Geography, 1990, Vol. 14, No 1, p. 24-56.

WAHL, H.W.; IWARSSON, S. \& OSWALD, F. Aging Well and the Environment: Toward an Integrative Model and Research Agenda for the Future. The Gerontologist, 2012, Vol. 52, № 3, p. 306-316.

WAHL, H.W. \& WEISMAN, G.D. Environmental gerontology at the beginning of new millennium: Reflections on Its historical, empirical, and theoretical development. The Gerontologist, 2003, Vol. 43, № 5, p. 612-627.

WILLIAMS, K. \& GREEN, S. Literature review of public space and local environments for the cross cutting review. Oxford: Oxford Brookes University, 2001. 